\title{
Reactive Power Optimization Simulation of Active Distribution Network Based on Particle Swarm Optimization
}

\author{
HaoZhang ${ }^{1}$, HongjuanLi ${ }^{2}$ and MinXu ${ }^{2, *}$ \\ ${ }^{1}$ State Grid Jiangxi Electric Power Company Shangrao Power Supply Branch, Shangrao 334000, China \\ ${ }^{2}$ School of Information Engineering, Nanchang University, Nanchang330031, China \\ ${ }^{*}$ Corresponding author
}

\begin{abstract}
The access of a large number of renewable energy makes the active distribution network become the inevitable trend of development, coordination of renewable distributed power and traditional reactive power compensation device can effectively achieve the active distribution network reactive power optimization. The simulation results of IEEE33 node system show that the mathematical model is correct and accurate for the reactive power optimization of the distribution network, and the particle swarm optimization algorithm can effectively search the global optimal.
\end{abstract}

Keywords-active distribution network; mathematical model; particle swarm algorithm; simulation

\section{INTRODUCTION}

The CIGRE C611 project team proposed the concept of active distribution network (ADN) in 2008, which clearly shows that ADN can manage the power flow by using a flexible network topology, in order to carry out active control and active management of local $\mathrm{DG}^{[1]}$. Distributed power supply can change the distribution network structure, while active distribution network can solve the problem of renewable distributed power consumption ${ }^{[2]}$. The purpose of reactive power optimization of active distribution network is to determine the output status of the reactive power compensation device over a period of time to improve the operating level of the active distribution network.

A large number of studies are based on the minimum loss ${ }^{[3]}$ as the objective function, prone to network loss optimal voltage deviation of the larger case, therefore, in order to avoid this situation, this paper establishes the mathematical model of reactive power optimization from the standpoint of reliability, and uses the voltage deviation as the objective function to solve the input capacity of the reactive power compensation device and the reactive power output of the PV grid by particle swarm optimization algorithm. Finally, the correctness and rationality of the model are verified by simulation of IEEE33 node distribution system.

\section{PARTICle SWARM Optimization Algorithm}

Particle Swarm Optimization (PSO) ${ }^{[4]}$ is a global random search algorithm proposed by Kennedy and Eberhart. PSO algorithm is an intelligent algorithm which is inspired by the foraging model of birds. It is widely used, The algorithm has the advantages of fast convergence, few parameters, simple algorithm and easy implementation.

\section{A. Basic Theory}

Particle swarm optimization is an intelligent optimization algorithm based on iterative model, which optimizes global optimality by updating individual optimal and group optimal search in iterations. Important information on the particles are: current position, historical optimal position and speed ${ }^{[5]}$. Assuming that the size of the particle population is $\mathrm{N}$, and each particle is searched in the D-dimensional space. The position vector of the particle $\mathrm{i}$ at the $\mathrm{t}$ times iteration $x_{i}^{t}=$ $\left[x_{i 1}^{t}, x_{i 2}^{t}, x_{i, 3}^{t}, \cdots, x_{i D}^{t}\right]$, speed vector $v_{i}^{t}=\left[v_{i 1}^{t}, v_{i 2}^{t}\right.$, $\left.\cdots, v_{i D}^{t}\right]$, particle individual optimal position $p_{i}^{t}=\left[p_{i 1}^{t}\right.$, $\left.p_{i 2}^{t}, \cdots p_{i D}^{t}\right]$,particle individual optimal position $p g_{i}^{t}=$ $\left[p g_{i 1}^{t}, p g_{i 2}^{t}, p g_{i 3}^{t}, \cdots p g_{i D}^{t}\right.$. The current position of the particle is a candidate solution corresponding to the optimization problem. The velocity of the particle represents the ability of the particle to traverse the solution space ${ }^{[6]}$, which can be dynamically adjusted according to the historical optimal position and the population history optimal position. According to the current position of the particle, the current fitness value of the particle can be calculated to evaluate the merits and demerits of the particle. Through the size of the fitness, the historical optimal position of the current particle can be determinedafter determining all the particles, position, through multiple iterations until the search for global optimizations.

\section{B. Algorithm Flow}

When the particle population is initialized, the value of each dimension variable is generated randomly according to the principle of not exceeding the range of values for each particle initial position and initial velocity. The particle determine the current flight speed of the vector by self-summary and learning from the individual in the group, to close their own historical advantages and the global optimal point of group; search the global optimal in the continuous iteration,and complete the optimization process.The specific process is as follows:

\section{1) Initialization}

Initialize the algorithm parameters. Such as: learning factor c, inertia weight $\mathrm{W}$, maximum iteration number MaxItr, search 
space dimension $\mathrm{D}$, population number $\mathrm{N}$ and other parameters.

\section{2) Initial position and velocity of each particle}

The population is initialized, and the group is an $\mathrm{N} \times \mathrm{D}$ matrix. Each dimension variable corresponding to the position vector and velocity vector is randomly generated within the range of values, as shown in equations (1) and (2).

$$
\begin{aligned}
& x_{i, d}=x_{d \min }+\left(x_{d \max }-x_{d \min }\right) \times \operatorname{rand}(1) \\
& v_{i, d}=v_{d \min }+\left(v_{d \max }-v_{d \min }\right) \times \operatorname{rand}(2)
\end{aligned}
$$

In the formula, $i=1,2,3, \ldots, \mathrm{N} ; d=1,2,3, \ldots, \mathrm{D}$; rand is the random number of the $(0,1)$ interval; The maximum and minimum values of the $d$ dimension are the maximum and minimum values of the velocity vector of particle $i$.

\section{3) Calculate the fitness}

The current fitness of the particle is calculated from the position of each particle, that is, the value corresponding to the objective function.

\section{4) Update the optimal}

Theupdateoftheoptimalfitnessincludetwoparts:updateofindi vidualoptimalvalueandgroupoptinalvalue.

Update individual optimal: compare the fitness of particle $i, f_{i}$ and its individual historical optimal value $f_{\text {ibest }}$, if better than the individual historical optimal value, the current fitness is the historical optimal value, namely:

$$
f_{\text {ibest }}=f_{i}(3)
$$

At the same time, the current position is also the historical optimal position of the particle,otherwise the optimal position and the optimal fitness update are not performed.

The optimal fitness of the population is better than that of the group. If the current fitness value is better than the group optimal value $F_{\text {best }}$, the current fitness is the group optimal value.

$$
F_{\text {best }}=f_{i}(4)
$$

At the same time, the current position is also the optimal position of the group,otherwise the optimal position and the optimal fitness update are not carried out.

\section{5) Update the particles}

The velocity and position of the particles are updated according to equations (5) and (6).

$$
\begin{array}{r}
v_{i d}^{t+1}=v_{i d}^{t}+c_{1} * r_{1}\left(p_{i d}^{t}-x_{i d}^{t}\right)+c_{2} * r_{2}\left(p g_{i d}^{t}-x_{i d}^{t}\right)(5) \\
x_{i d}^{t+1}=x_{i d}^{t}+v_{i d}^{t+1}(6)
\end{array}
$$

In the formula, $c_{1}$ and $c_{2}$ are acceleration factor, $t$ is the current number of iterations, $r_{1}$ and $r_{2}$ are random numbers in the $(0,1)$ interval.

\section{6) Judgment of termination condition}

Judgment of the termination condition, if the termination condition is judged and the case is not satisfied, repeat the third step until the termination condition is satisfied.

\section{ACTIVE POWER DISTRIBUTION OPTIMIZATION}

Considering the influence of the access of the distributed power supply in the active distribution network on the network structure of the distribution network ${ }^{[7]}$, it is necessary to realize the coordinated control of the distributed power supply and the reactive power compensation device. The active distribution network reactive power optimization can help ensure that the node voltage is in the normal range of operation, and improve the voltage quality.

\section{A. Objective Function}

Under certain constraints, active distribution network reactive power optimization can achieve the optimal objective function, making the node voltage in the normal range of operation and improving the active distribution network operation level, through the coordination of distributed power supply and traditional reactive power compensation device. The objective function :

$$
F_{1}(x)=\sum_{i=1}^{T} \frac{\left|\sqrt{\left(V_{j}(x)-\Delta V_{i j}(x)\right)^{2}+\left(\delta V_{i j}(x)\right)^{2}}-V_{N}\right|}{V_{N}}(7)
$$

In the formula, $i=1,2,3, \ldots, \mathrm{T}, \mathrm{T}$ is the total number of distribution network nodes, $V_{N}$ is rated voltage, $i, j$ is the first node and end node of the branch respectively, $\Delta V_{i j}$ is the vertical component of voltage drop of branch $i j, \quad \delta V_{i j}$ is the horizontal component of voltage drop of branch $i j, x$ is the control variable which is the output of the distributed power and the reactive power compensation device in this paper.

\section{B. Restrictions}

\section{1) Equality constraints}

Power flow restriction including DG

$$
\left\{\begin{array}{l}
P_{i}+P_{D G i}=P_{D i}+U_{i} \sum_{j=1}^{N_{b}} U_{j}\left(G_{i j} \cos \delta_{i j}+B_{i j} \sin \theta_{i j}\right) \\
Q_{i}+Q_{D G i}=Q_{D i}+U_{i} \sum_{j=1}^{N_{b}} U_{j}\left(G_{i j} \cos \delta_{i j}-B_{i j} \sin \theta_{i j}\right)
\end{array}\right.
$$

In the formula, $P_{i} 、 Q_{i}$ is the active power and reactive power input to the node $i, P_{D G i} 、 Q_{D G i}$ is the active power and reactive power input to the node $i$ by DG, $P_{D i} 、 Q_{D i}$ is the active power and reactive power at the node $i, U_{i} 、 U_{j}$ is voltage of node $i$ and node $j, G_{i j}, B i j$ and $\delta_{i j}, \theta_{i j}$ is the conductivity, charge and phase angle difference of node $i$ and node $j$, which are related to control variables.

\section{2) Inequality constraints}

$$
Q_{\text {imin }} \leq Q_{\mathrm{i}} \leq Q_{\text {imax }}
$$




$$
U_{\text {imin }} \leq U_{i} \leq U_{\text {imax }}
$$

In the formula, $Q_{\text {imax }}$, $Q_{\text {imin }}$ is the maximum reactive power output and minimum reactive power output of the capacitor $i$. In formula (10), $U_{\mathrm{imax}}, U_{\mathrm{imin}}$ is the voltage upper limits and lower limits of node $i$.

\section{Specific steps}

The particle swarm algorithm is applied to the active distribution network reactive power optimization. The specific process is as follows:

1) Initialization of algorithm parameters: The maximum number of iterations, MaxItr $=100$, the spatial dimension $\mathrm{D}=5$, the number of initialized groups $\mathrm{N}=50$ and other related algorithm parameters.

2) According to the principle of the algorithm, the initial position and velocity of each particle are randomly generated according to the range of variables in equations (1) and (2).

3) Calculate the fitness of each particle, that is, the objective function voltage deviation.

4) Update. For the individual, update the history of the best fitness and location, while for the group , update the best fitness and location.

5) Update the position variable and velocity variable of the particle, that is, updating the current search of the control variable, and determining the position of the next iteration based on the current position and velocity.

6) Determine the termination of the conditions, or repeat the third step, until the termination of the iteration to get the optimal optimization strategy.

The specific flow chart shown in Figure 1:

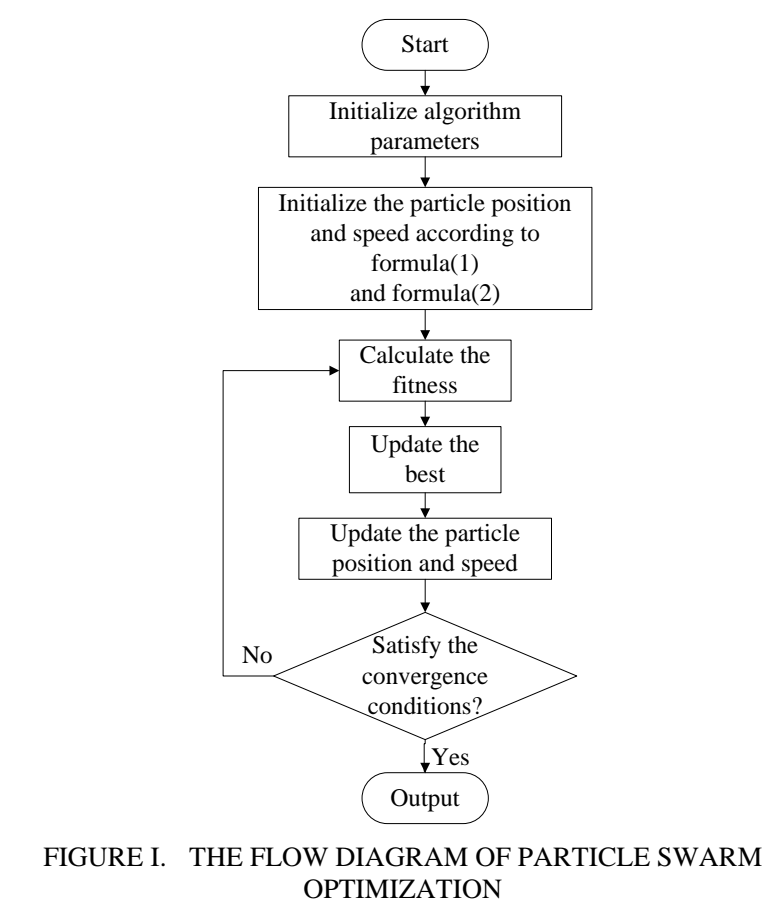

FIGURE I. THE FLOW DIAGRAM OF PARTICLE SWARM OPTIMIZATION

\section{CASE STUDY}

The examples used in this paper are based on the standard IEEE33 node modification. Standard IEEE33 node system network has 32 branches, a power supply network. On this basis, 18 nodes access to photovoltaic power generation, the current active output $160 \mathrm{~kW}$, Photovoltaic inverter maximum capacity 200kVA, the minimum power factor 0.8 , reactive power adjustment range $0-120 \mathrm{kvar}$; node $6,11,25,33$ nodes access capacitor, reactive power compensation range 0-1200kvar, 24 nodes access to the fuel cell, the current active output $160 \mathrm{~kW}$, reactive power $120 \mathrm{kvar}, 31$ node wind power active $300 \mathrm{~kW}$, reactive power output $200 \mathrm{kvar}$.

Based on the above data, the voltage of each node is within the normal range. In order to verify the correctnessof particle swarm algorithm, the active power of the node 32 is set to 0.1 (per unit value), and the reactive power is set to 0.1 (per unit value) . Each node voltage is shown in Table 1 in the following two cases: no reactive compensation, reactive power optimization by particle swarm algorithm. Particle swarm algorithm proposed control strategy is shown in Table 2. 
TABLE I. NODAL VOLTAGE

\begin{tabular}{|c|c|c|c|c|c|c|c|c|}
\hline Node & $\begin{array}{c}\text { Voltage } \\
\text { before } \\
\text { compensation }\end{array}$ & $\begin{array}{c}\text { Voltage at } \\
\text { compensation } \\
\text { point by PSO }\end{array}$ & Node & $\begin{array}{c}\text { Voltage } \\
\text { before } \\
\text { compensation }\end{array}$ & $\begin{array}{c}\text { Voltage at } \\
\text { compensation } \\
\text { point by PSO }\end{array}$ & Node & $\begin{array}{c}\text { Voltage } \\
\text { before } \\
\text { compensation }\end{array}$ & $\begin{array}{c}\text { Voltage at } \\
\text { compensation } \\
\text { point by PSO }\end{array}$ \\
\hline 1 & 1.0500 & 1.0500 & 12 & 0.9719 & 1.0033 & 23 & 1.0281 & 1.0330 \\
\hline 2 & 1.0461 & 1.0475 & 13 & 0.9676 & 1.0000 & 24 & 1.0231 & 1.0280 \\
\hline 3 & 1.0308 & 1.0357 & 14 & 0.9660 & 0.9990 & 25 & 1.0200 & 1.0249 \\
\hline 4 & 1.0219 & 1.0294 & 15 & 0.9653 & 0.9987 & 26 & 0.9868 & 1.0074 \\
\hline 5 & 1.0129 & 1.0231 & 16 & 0.9648 & 0.9986 & 27 & 0.9827 & 1.0045 \\
\hline 6 & 0.9899 & 1.0095 & 17 & 0.9642 & 0.9993 & 28 & 0.9635 & 0.9932 \\
\hline 7 & 0.9868 & 1.0093 & 18 & 0.9642 & 0.9998 & 29 & 0.9495 & 0.9852 \\
\hline 8 & 0.9829 & 1.0066 & 19 & 1.0438 & 1.0470 & 30 & 0.9431 & 0.9810 \\
\hline 9 & 0.9780 & 1.0053 & 20 & 1.0247 & 1.0436 & 31 & 0.9312 & 0.9770 \\
\hline 10 & 0.9736 & 1.0044 & 21 & 1.0240 & 1.0429 & 32 & 0.9264 & 0.9753 \\
\hline 11 & 0.9729 & 1.0042 & 22 & 1.0234 & 1.0423 & 33 & 0.9262 & 0.9791 \\
\hline
\end{tabular}

TABLE II. CONTROL STRATEGY

\begin{tabular}{|c|c|}
\hline $\begin{array}{c}\text { Reactive power } \\
\text { compensation device }\end{array}$ & $\begin{array}{c}\text { Reactive power output by } \\
\text { PSOQ/kvar }\end{array}$ \\
\hline PV (Node18) & 120 \\
\hline capacitor1 (Node6) & 0 \\
\hline capacitor2 (Node11) & 634 \\
\hline capacitor3 (Node25) & 1200 \\
\hline capacitor4 (Node33) & \\
\hline
\end{tabular}

From the above data, in the case which the voltages of the nodes 30, 31, 32, 33 are low, after the particle swarm algorithm is optimized for reactive power,the nodes $30,31,32,33$ voltage is in the normal range, while the system most of the node voltage has reached the rated value and achieve the goal of reactive power optimization.

\section{RESULT}

Taking the reactive power optimization of the active distribution network as the research object, the distributed power source and the traditional reactive power compensation device are taken as the control variables, and the reactive power optimization mathematical model with the voltage deviation as the objective function is established. The mathematical model avoids the situation of overrun of voltage and lowest network loss in the traditional distribution network with the network loss as the objective function. The results of IEEE33 node system show that the algorithm is feasible and the correctness of the reactive power optimization model is validated by applying the particle swarm algorithm to the reactive power optimization of active distribution network.

\section{REFERENCES}

[1] Bo ZHAO, CaishengWANG, andJinhuiZHOU. "Present and Future Development Trend of Active Distribution Network", Automation of Electric Power System, 2014, vol. 38, pp. 125-135.

[2] ChunjuGUO. "The Effect of Power System Connected with Mass Distribution Generation”, Shanghai Jiao Tong University, 2013.
[3] KangGUO, YuqinXU, and LiZHANG. "Reactive Power Optimization of Distribution Network Considering PV Station Randon Output”, Power System Protection and Control, 2012, vol. 40, pp. 53-58.

[4] YanminBAI. "Particle Swarm Optimization and Its Application", Lanzhou Jiaotong University, 2013.

[5] JianhuaLIU. "The Research of Basic Theory and Improvement on Particle Swarm Optimization”. Central South University, 2009.

[6] XuewenXIA, JingnanLIU, and KefuGAO. "Particle Swarm Optimization Algorithm with Reverse-Learning and Local-Learning Behavior”, Chinese Journal of Computers, 2015, vol. 38, pp. 1397-1407.

[7] JianchengYU, FujianCHI, and KeXU. "Analysis of the Impact of Distribution Generation on Power Grid". Proceedings of the CSU-EPSA, 2012, vol. 24, pp. 138-141. 